References:

[1] Dessein PH, Joffe BI, Metz RM, Millar DL, Lawson M, Stanwix AE. Autonomic dysfunction in systemic sclerosis: sympathetic overactivity and instability. The American journal of medicine. 1992;93(2):143-50.

Acknowledgments: None

Disclosure of Interests: None declared

DOI: 10.1136/annrheumdis-2020-eular.6149

\section{AB0614 METHOTREXATE DOESN'T LOWER THE RISK OF DEVELOPING INTERSTITIAL LUNG DISEASE IN PATIENTS WITH IDIOPATHIC INFLAMMATORY MYOPATHIES WITH JO-1 ANTIBODIES.}

P. Szczęsny ${ }^{1}$, K. Swierkocka ${ }^{1}$, A. Felis-Giemza ${ }^{1}$, M. Olesińska ${ }^{1} .{ }^{1}$ National Institute of Geriatrics, Rheumatology and Rehabilitation, Department of Connective Tissue Diseases, Warsaw, Poland

Background: In patients with idiopathic inflammatory myopathies (IIM) most commonly found autoantibody against histidyl-tRNA synthetase (anti-Jo-1) is associated with development of interstitial lung disease (ILD), which has been recognized as a serious mortality factor.

Objectives: To assess if methotrexate as an initial steroid sparing agent lowers the risk of developing ILD in anti-Jo-1 positive patients diagnosed with IIM.

Methods: Medical records of IIM patients treated in a referral clinic in capital city of Poland between 2008 and 2018 were reviewed. Inclusion criteria were: fulfillment of ACR/EULAR 2017 classification criteria for IIM, positivity of anti-Jo-1 antibodies in the EUROLINE test, introduction of corticosteroids equivalent to $\geq 0,5 \mathrm{mg}$ of prednisone. Exclusion criteria: insufficient data on disease course, history of IIM $<18$ months.

Results: 29 patients were included for this analysis. ILD was present at the onset in $52 \%(\mathrm{n}: 15)$ patients. Other 14 patients were treated initially with corticosteroids $\geq 0,5 \mathrm{mg} / \mathrm{kg}$ along with methotrexate up to $25 \mathrm{mg} /$ week. In all 14 patients methotrexate was well tolerated and led to successful reduction of steroid dose. However, ILD attributed to the primary disease appeared in follow up in $50 \%(n: 7)$ of these patients (medium 36 months), which resulted in alteration of treatment. In 7 patients ILD didn't develop.

Conclusion: Our study shows that methotrexate in dose up to $25 \mathrm{mg} /$ week doesn't lower the risk of developing ILD in Jo-1 positive IIM patients in the long term suggesting that other medication should be used as a first line treatment for this group.

References:

[1] Factors affecting survivorship in polymyositis: a life-table study of 124 patients. Medsger TA, Robinson H, Masi AT. Arthritis Rheum 1971;14: 249-58

[2] (2018). Treatment of adult idiopathic inflammatory myopathies with conventional immunosuppressive drugs. Keyßer, G., Zierz, S., \& Kornhuber, M. Z Rheumatol. 2019;78(2):183-189.

[3] 2017 European League Against Rheumatism/American College of Rheumatology classification criteria for adult and juvenile idiopathic inflammatory myopathies and their major subgroups Lundberg IE, Tjärnlund A, Bottai M, et al. Ann Rheum Dis. 2017;76(12):1955-1964.

Disclosure of Interests: None declared

DOI: 10.1136/annrheumdis-2020-eular.5028

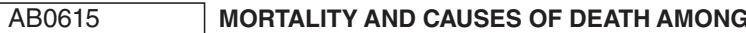 ROMANIAN SYSTEMIC SCLEROSIS PATIENTS}

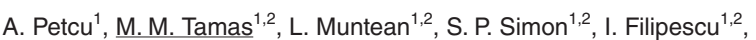
L. Damian", D. C. Leucuta ${ }^{3}$, S. Rednic ${ }^{1,2}{ }^{2}$ "Iuliu Hatieganu"University of Medicine and Pharmacy, Rheumatology, Cluj-Napoca, Romania; ${ }^{2}$ Emergency County Clinical Hospital, Cluj-Napoca, Romania; "' "Iuliu Hatieganu" University of Medicine and Pharmacy, Department of Medical Informatics and Biostatistics, Cluj- Napoca, Romania

Background: Systemic sclerosis (SSc) is associated with an increased risk of death compared to the general population. Survival in SSc patients has improved significantly over the last 20 years with a decrease in renal involvement as a cause of early death and an increase in death caused by cardiopulmonary involvement $(1,2)$. Causes of death in SSc patients have not been described in a Romanian cohort so far.

Objectives: To study the causes of death in patients with SSc prospectively followed-up from 2002 to 2018 in a single tertiary centre from Romania.

Methods: The cohort consists of 197 patients who fulfill the American College of Rheumatology /EULAR 2013 criteria for SSc. We examined the data of patients who had died during follow up. Patients were reviewed at least twice a year and the cause of death was classified as SSc-related or nonSSc-related.
SSc-related deaths were then attributed to the major organ involved. A univariate Cox proportional hazard $(\mathrm{PH})$ regression was used to examine the association between each variable and mortality. Variables reported in the literature to associate with mortality were considered in the multiple $\mathrm{Cox} \mathrm{PH}$ regression model.

Results: Of 197 SSc patients ( $87.8 \%$ females), $47.7 \%$ had diffuse SSc and $52.2 \%$ had limited SSc. The mean age at diagnosis was 47 (SD 12) years and mean follow up duration was 6.75 years. There were 41 deaths $(20.8 \%)$ Survival rate was substantially lower in men $(P<0.003)$. The mean age at the time of death in those with diffuse SSc was lower compared to limited SSc (55.8 years vs 68.7 years). Sixty percent of deaths were SSc-related (pulmonary cause[ $n=11]$, cardiac cause[ $n=6]$, gastrointestinal involvement [GI, $n=3]$, renal crisis [n=2] and others $[n=4])$. Deaths no related to SSc were associated with cancer and infections. Age at onset of Raynaud phenomenon [HR 1.05], male gender [HR 3.41], diffuse SSc [HR 0.48], presence of tendon friction rub [HR 4.54], digital ulceration [HR 3], esophagitis [HR 2.07] and cardiovascular involvement [HR 3.68], use of corticosteroids[HR 2.13] and cyclophosphamide [HR 2.02] were associated with poor prognosis in multivariate analysis.

Conclusion: In our cohort the main causes of death were lung and cardiovascular involvement. Deaths occurred early after the onset of the disease and the survival rate was significantly reduced among men. Multivariate analysis showed that age at onset of Raynaud phenomenon, male gender, diffuse disease form presence of tendon friction rub, digital ulceration, esophagitis and cardiovascular history, use of corticosteroids and cyclophosphamide were independently associated with mortality.

References:

[1] Tyndall AJ, Bannert B, Vonk M, Airo P, Cozzi F, Carreira PE, et al. Causes and risk factors for death in systemic sclerosis: a study from the EULAR Scleroderma Trials and Research (EUSTAR) database. Ann Rheum Dis. 2010 Oct;69(10):1809-15.

[2] Rubio-Rivas M, Royo C, Simeon CP, Corbella X, Fonollosa V. Mortality and survival in systemic sclerosis: systematic review and meta-analysis. Semin Arthritis Rheum. 2014 Oct;44(2):208-19.

Disclosure of Interests: None declared

DOI: 10.1136/annrheumdis-2020-eular.5438

\section{AB0616 REDUCED BONE MINERAL DENSITY IN PATIENTS WITH IDIOPATHIC INFLAMMATORY MYOPATHIES: A LONGITUDINAL STUDY}

Y. K. Tang ${ }^{1}$, H. So ${ }^{1}$, T. L. V. Wong ${ }^{1}$, H. T. Pang ${ }^{1}$, V. Lao ${ }^{1}$, M. L. Yip ${ }^{2} .{ }^{1}$ Kwong Wah Hospital, Kowloon, Hong Kong (SAR); ${ }^{2}$ TWGHs Integrated Diagnostic and Medical Centre, Kowloon, Hong Kong (SAR)

Background: Reduced bone mineral density (BMD) leads to fragility fracture which is associated with a significant morbidity and excess mortality $[1,2]$ Patients with idiopathic inflammatory myopathies (IIM) should be at a heightened risk of reduced BMD as a result of the systemic inflammation, reduced mobility and corticosteroid use [3]. A previous cross-sectional study demonstrated a high prevalence of osteoporosis (23.7\%) and osteopenia (47.4\%) in a cohort of IIM patients [4]. However, longitudinal data are lacking.

Objectives: To assess the BMD of IIM patients longitudinally and to investigate the factors associated with accelerated bone loss.

Methods: This is a single centered observational study. Existing adult Chinese patients with IIMs who had serial BMD measurements done were recruited. The diagnosis of IIMs was based on the Bohan and Peter's criteria with definite or probable cases being included [5]. Patients with clinically amyopathic disease must have the typical Gottron's papules or heliotrope rash as determined by rheumatologists or dermatologists, and with no symptoms or signs of muscle involvement according to Sontheimer [6]. BMD was measured by dual energy $\mathrm{X}$-ray absorptiometry (DEXA). Clinical variables thought to be associated with bone health were documented.

Results: All together 28 patients were studied. The mean age of the patients at disease onset was 46.1 years (S.D. 12.2). There was a female predominance $(92.9 \%)$. The subgroups of IIMs were: dermatomyositis $(39.3 \%)$, polymyositis $(25.3 \%)$, clinically amyopathic dermatomyositis $(21.4 \%)$ and immune mediated necrotising myopathy $(14.3 \%)$. Only a minority of the patients smoked $(7.1 \%)$ and none of them drinks regularly. About one fifth of the patients were underweight. All patients have been exposed to systemic corticosteroid, while $82.1 \%$ of them were still on it between the two scans with $32.1 \%$ even on high dose $(>0.5 \mathrm{mg}$ prednisolone $/ \mathrm{kg} / \mathrm{day}$ ). Three out of the 28 patients $(10.7 \%)$ was found to be osteoporotic at baseline and 17 patients $(60.7 \%)$ were osteopenic Follow-up DEXAs were performed mostly 5 to 10 years after the initial scan Despite 8 patients $(28.6 \%)$ were given active anti-osteoporotic medications, the bone health deteriorated significantly. The mean baseline neck of femor BMD dropped from 0.711 to $0.657 \mathrm{~g} / \mathrm{cm} 2(p=0.042)$ on follow-up, while the total 